\title{
Avaliação de Obras de Arte Especiais da Cidade de Brasília pela Metodologia do Grau de Deterioração Estrutural da Universidade de Brasília
}

\author{
G. Berbert-Born ${ }^{1 *}$, J.G. Monteiro ${ }^{1}$, A. Nascimento Filho ${ }^{1}$, \\ L. Chaves ${ }^{1}$, M. E. Pereira ${ }^{1}$, M. H. Oliveira ${ }^{1}$ \\ *Autor de Contacto: guiborn.eng@gmail.com \\ ${ }^{1}$ Departamento de Engenharia Civil e Ambiental, Faculdade de Tecnologia, Universidade de Brasília - UnB, Brasil
}

\begin{abstract}
RESUMO
Viadutos e pontes são estruturas essenciais para garantir a continuidade das estradas quando se cruzam com obstáculos. No Brasil, o concreto armado é o material mais utilizado nessas estruturas devido à sua trabalhabilidade e resistência. O monitoramento constante é, entretanto, necessário para garantir o desempenho e evitar o aparecimento de manifestações patológicas. Nos anos de 2020 e 2021, foram identificados 356 viadutos e pontes no Distrito Federal. Este trabalho tem como objetivo mostrar os resultados da inspeção de 168 dessas estruturas utilizando uma metodologia que classifica o seu grau de deterioração, recorrendo a formulações baseadas em inspeções visuais. A classificação do grau de deterioração estrutural permitiu estabelecer um panorama técnico e forneceu dados para a criação de uma base de dados com informações importantes sobre o estado de conservação e integridade estrutural dessas pontes e viadutos.
\end{abstract}

Palavras Chaves: Viadutos e pontes; OAEs; Concreto Armado; Inspeção; Manifestações Patológicas. 


\begin{abstract}
Viaducts and bridges are essential structures to ensure the continuity of roads when they intersect obstacles. In Brazil, reinforced concrete is the most common material used in such structures due to its workability and resistance. Constant monitoring is, however, necessary to guarantee the performance and to avoid the appearance of pathological manifestations. In the years of 2020 and 2021, a total of 356 viaducts and bridges were identified in the Federal District. This work objectives to show the results of the inspection of 168 of those structures using a methodology which classifies their degree of deterioration, resorting to formulations based on visual inspections. The classification of the degree of structural deterioration made it possible to establish a technical overview and provided data for the creation of a database with important information on the state of conservation and structural integrity of such bridges and viaducts.
\end{abstract}

Keywords: Viaducts and bridges; Concrete; Pathological Manifestations; Degree of deterioration; Maintenance.

\title{
RESUMEN
}

Los viaductos y puentes son medios esenciales para asegurar la continuidad de las vías cuando encuentran obstáculos. El hormigón es el material más utilizado en estas estructuras por su trabajabilidad y resistencia, sin embargo, es necesario un monitoreo constante para asegurar su desempeño y evitar el desarrollo de patologías. En los años 2020 y 2021, un total de 356 viaductos y puentes fueran levantadas en el Distrito Federal. Este trabajo tiene como objetivo presentar los resultados de la inspección de 168 estructuras utilizando una metodología que clasifica el grado de deterioro de las estructuras, utilizando formulaciones basadas en inspecciones visuales. La clasificación del grado de deterioro estructural proporcionó una descripción técnica y generó datos para la creación de uma base de datos com información de gran importância sobre el estado de conservación e integridade estructural de estas Obras de Arte Especiales (OAEs).

Palabras clave: Viaductos y puentes; OAEs; Hormigón armado; Inspección; Manifestaciones patológicas.

\section{INTRODUÇÃO}

As Obras de Arte Especiais (OAEs) são estruturas que têm a função de ligar pontos separados por obstáculos naturais ou artificiais. Algumas OAEs são compostas por pontes (quando construídas sobre cursos d'água) e viadutos (quando construídas sobre avenidas ou vales secos). Essas construções estão submetidas as ações externas provenientes de carregamento gravitacional estético, carregamentos móveis produzidos por veículos, ações dinâmicas devido ao vento, exposição à água, variações de temperatura, retração e fluência no caso de estruturas de concreto armado. É notório que as OAEs são afetadas pela degradação dos materiais, fadiga, corrosão, ausência ou falha de manutenção, pelo incremento de cargas, entre outros fatores.

As estruturas de concreto armado devem atender os requisitos de qualidade, classificados em capacidade resistente, desempenho em serviço e durabilidade. Nota-se que a capacidade resistente está relacionada à segurança à ruptura da estrutura. $\mathrm{O}$ desempenho em serviço é evidenciado pela capacidade da estrutura atender as condições de utilização durante sua vida útil. A durabilidade, por sua vez, está relacionada ao grau de exposição da estrutura a fatores ambientais ao longo do tempo e à boa conduta de utilização dos usuários. 
Brasília, a capital do Brasil inaugurada em 1960, evidenciou aspectos preocupantes nos últimos anos em função das auditorias realizadas por órgãos de fiscalização, como a realizada pelo Tribunal de Contas do Distrito Federal - TCDF em 2012. A auditoria apontou 7 viadutos cuja manutenção deveria ser realizada em caráter de urgência. Em fevereiro do ano de 2018 ocorreu o desabamento de parte da porção central do viaduto sobre a Galeria dos Estados do Eixo Rodoviário da cidade de Brasília, sendo este um dos viadutos apontados na auditoria do TCDF. A necessidade de desvios no trânsito de uma das principais vias da cidade de Brasíla, devido ao isolamento para reabilitação do trecho desabado do viaduto, expôs prejuízo significativo ao erário e ao sistema de transporte dessa região. Fortuitamente, o episódio não foi marcado por perda de vidas humanas, ainda que a estrutura fosse utilizada intensamente pelos usuários do transporte metroviário da cidade.

De acordo com Mitre (2005), manifestações patológicas são os sinais visualmente aparentes ou identificáveis com o uso de instrumentos de algum processo de deterioração nas edificações. A descrição dos principais sintomas, locais e formas de ocorrência, bem como da gravidade e extensão são fundamentais para o diagnóstico, avaliação e prognóstico de uma obra deteriorada. Muitas vezes, somente a observação pericial dos sintomas e pequenos testes de campo são suficientes para que um especialista realize uma análise crítica que conduza ao correto diagnóstico do problema e demais etapas, possibilitando uma conduta corretiva ou preventiva eficaz.

Diante do exposto, pode-se identificar, diagnosticar e solucionar os problemas antes que atinjam proporções graves ou que resultem em custos financeiros e sociais elevados de recuperação. Por esse viés, o acompanhamento periódico do estado das OAEs, com base em vistorias e inspeções detalhadas, executadas conforme um conjunto de procedimentos padronizados e tecnicamente adequados, é o modo mais eficaz de subsidiar uma política de manutenção corretiva e preventiva de OAEs.

Inspeções detalhadas permitem identificar as principais manifestações patológicas atuantes nas estruturas em análise. Por meio destas, é possível estabelecer planos de manutenção, a fim de que se possa atender os requisitos mínimos de capacidade de carga, segurança e conforto, permitindo a ampliação do período de preservação e de serviço das edificações. A avaliação visual pode ser utilizada como método de inspeção, bem como a realização de ensaios não destrutivos dos elementos das OAEs.

Diante da importância das OAEs do Distrito Federal, foram promovidas inspeções e avaliações de 168 pontes e viadutos presentes nessa região. Este artigo expõe a realização dessas ações pelos autores advindos da Universidade de Brasília (UnB). Tomando como referência inicial a Metodologia Klein et al. (1991), cujo trabalho propôs a criação de um método que visa a padronização da execução de vistorias em OAEs por meio da separação dos elementos construtivos em famílias, a proposta de avaliação das pontes e viadutos deste trabalho tem por base a metodologia do Grau de Deterioração Estrutural (GDE/UnB), proposta por Castro (1994) e atualizada por diferentes pesquisadores da Universidade de Brasília nos últimos anos.

A metodologia do Grau de Deterioração Estrutural (GDE/UnB) corresponde a um método que faz uso de equações para análise do grau de deterioração estrutural, além de permitir a avaliação, separadamente, de cada elemento da estrutura. A metodologia GDE/UnB pode ser utilizada como uma ferramenta de apoio à decisão dos gestores públicos e privados, permitindo identificar as principais manifestações patológicas que afetam as estruturas, podendo fornecer subsídios para que órgãos públicos e empresas estimem e promovam planos de manutenções preventivas e corretivas. Desenvolvida por Castro (1994) para quantificar o grau de deterioração em estruturas de concreto armado convencionais, a metodologia evoluiu por meio das pesquisas de Lopes (1998), Boldo (2002), Fonseca (2007), Euqueres (2011) e Verly (2015). As adaptações evidenciadas por esses autores incidem nas formulações e parâmetros utilizados pelos autores no desenvolvimento das inspeções abordadas no presente trabalho. 
A base da metodologia se dá no particionamento das edificações inspecionadas, tomando como medida de separação as características estruturais dos elementos, em grupos denominados famílias de elementos. Cada família detém uma matriz com possíveis manifestações de danos. Cada possibilidade de manifestação acompanha um valor de Fator de Ponderação do Dano $\left(\mathrm{F}_{\mathrm{p}}\right)$ com o objetivo de quantificar a relevância de um determinado dano em função dos quesitos de estética, funcionalidade e segurança. Com a inspeção visual, o responsável técnico deve atribuir o Fator de Intensidade do Dano $\left(\mathrm{F}_{\mathrm{i}}\right)$, classificando assim a gravidade do dano. A fase seguinte da metodologia diz respeito ao Grau do Dano $(D)$, fator introduzido na metodologia para quantificar a manifestação de cada dano no elemento com base na evolução temporal da deterioração do concreto. Por meio desses dados, é possível calcular o Grau de Deterioração de um Elemento $\left(\mathrm{G}_{\mathrm{de}}\right)$ e, em sequência, determinar o Grau de Deterioração de uma Família de Elementos $\left(\mathrm{G}_{\mathrm{df}}\right)$ dado pela média aritmética entre os graus de deterioração dos elementos componentes da família.

Cada família de elementos em análise deve possuir um fator de relevância estrutural $\left(\mathrm{F}_{\mathrm{r}}\right)$, de modo a considerar a importância relativa das diversas famílias de elementos no conjunto de partições da obra. O parâmetro final de análise é definido como o Grau de Deterioração da Estrutura $\left(\mathrm{G}_{\mathrm{d}}\right)$ composto pela média ponderada dos graus de deterioração das famílias de elementos e seus respectivos fatores de relevância estrutural. Com a obtenção desse valor, é possível classificar a estrutura analisada em níveis que variam de estado baixo a crítico de deterioração, de acordo com a separação feita por Euqueres (2011). A Figura 1 mostra o fluxograma adaptado de Castro (1994) proposto para a metodologia GDE/UnB e suas etapas de avaliação.

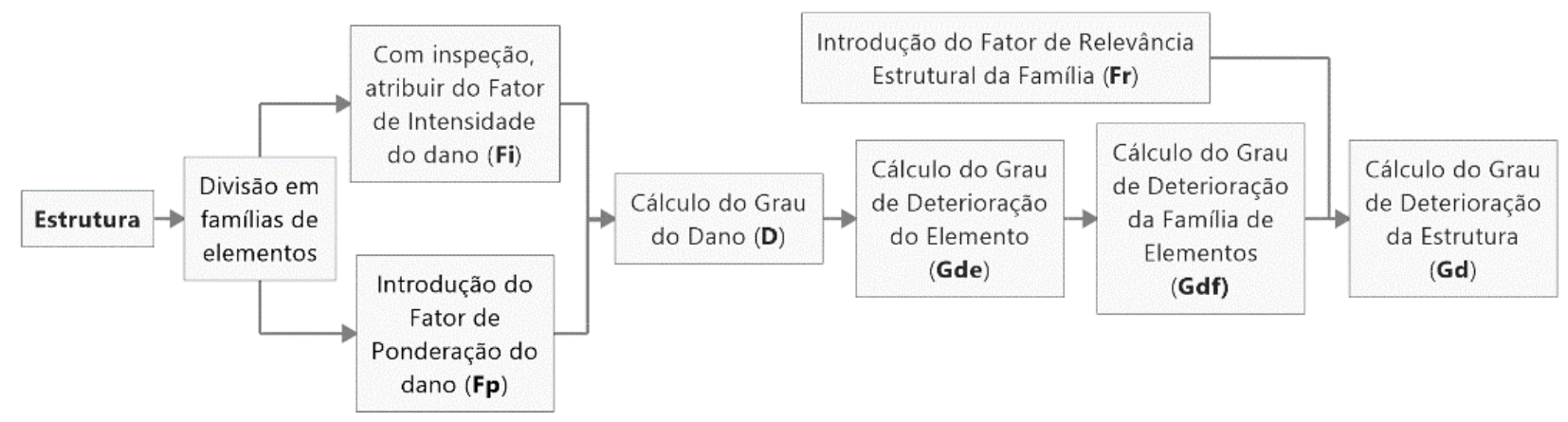

Figura 1 - Fluxograma da metodologia GDE/UnB (CASTRO,1994 - adaptado)

O acompanhamento da degradação das OAEs da região de Brasília é ação fundamental para a preservação do patrimônio histórico da humanidade revelado pela cidade. A exposição direta aos agentes ambientais e o regime dinâmico de algumas solicitações na estrutura aceleram a evolução das manifestações patológicas enfrentadas por essas estruturas. Este trabalho expõe o levantamento das condições estruturais e a avaliação de 168 pontes e viadutos do Distrito Federal, compondo o banco de dados do Sistema de Gestão de Obras de Arte Especiais do Distrito Federal, SiGOA-DF.

\section{PROCEDIMENTO}

A metodologia GDE/UnB foi aplicada considerando a especificidade da aplicação da mesma em OAEs bem como as alterações e modificações realizadas ao longo dos estudos de diversos autores, responsáveis pela criação e desenvolvimento da metodologia.

\subsection{Abordagem da Metodologia GDE/UnB}

A metodologia GDE/UnB foi aplicada seguindo suas últimas revisões e adaptações da literatura técnica. Sendo assim, a aplicação foi feita levando em conta o Manual de Aplicação da Metodologia GDE/UnB a OAEs proposto por Verly (2015), considerando o dano de abrasão proposto por Lauria 
(2018) e utilizando constantemente o catálogo de danos propostos por Pavoni (2019). Além disso, as análises foram realizadas através de inspeções visuais com registros fotográficos dos danos encontrados, logo não foram considerados os danos de carbonatação e contaminação do concreto por cloretos, devido à necessidade de ensaios para a avaliação destas manifestações patológicas. Os cálculos da metodologia aplicada foram feitos utilizando a equações (1) e (2) para definição do grau de dano em cada caso e o grau de deterioração do elemento foi calculado em acordo com a equações (3).

$D=0,8 \cdot F_{i} \cdot F_{p}$, para $F_{i} \leq 2$
$D=\left(12 \cdot F_{i}-28\right) \cdot F_{p}$, para $F_{i} \geq 3$

$G_{d e}=D_{\text {máx }} \cdot\left(1+\frac{\sum_{i=1}^{m} D_{(i)}-D_{\text {máx }}}{\sum_{i=1}^{m} D_{(i)}}\right)$

A partir dos fatores de relevância das famílias, o cálculo prossegue para o grau de deterioração da família na equação (4) e, em seguida, para o grau de deterioração estrutural da obra analisada na equação (5).

$$
\begin{gathered}
G_{d f}=G_{d e m a x} \cdot\left(\sqrt{1+\frac{\sum_{i=1}^{m} G_{d e(i)}-G_{d e m a x}}{\sum_{i=1}^{m} G_{d e(i)}}}\right) \\
G_{d, \bmod }=\frac{K_{\max }}{7,07} \cdot \sqrt{1+\frac{\left(\sum_{i=1}^{k} K_{i}\right)-K_{\max }}{\sum_{i=1}^{k} K_{i}}}
\end{gathered}
$$

Foram utilizados os mesmos fatores de relevância estrutural das famílias de elementos da estrutura propostos na ficha de inspeção usada por Lauria (2018), porém excluiu-se o elemento pista de rolamento e foram incluídos os elementos pilares, pilares-parede, longarina e transversina, usando os fatores de relevância de acordo com Verly (2015). Seguem os valores de Fr elencados para cada família.

- Guarda-rodas

- Cortina, Contenção, Transversina

- Tabuleiro

- Pilar, Pilar-Parede, Longarina

$$
\begin{aligned}
& F_{r}=1 \\
& F_{r}=3 \\
& F_{r}=4 \\
& F_{r}=5
\end{aligned}
$$

Com isso, se procedeu com a análise utilizando a metodologia para os viadutos selecionados calculando dois valores de grau de deterioração da estrutura (Gd* e Gd), isto é, desconsiderando e considerando, respectivamente, os valores de grau de deterioração de elementos (Gde) menores que 15.

Vale ressaltar que, devido as variações na distribuição e seleção de elementos estruturais em cada Obra de Artes Especial analisada, foi necessário realizar divisões específicas para diferentes 
estruturas, sendo isso feito em conformidade com os princípios utilizados por Lauria (2018) e Pavoni (2019), visando uma melhor discretizarão das estruturas. O método de divisão se baseia na determinação de referências cardeais para identificação dos elementos.

\subsection{Viadutos selecionados para inspeção}

Para a seleção das OAEs a serem inspecionadas, inicialmente, foi feito uma divisão da área correspondente a região do Distrito Federal, separada por uma malha de 10 x 10, gerando 100 quadrados iguais, dividindo todo o Distrito Federal, conforme mostra a Figura 2.

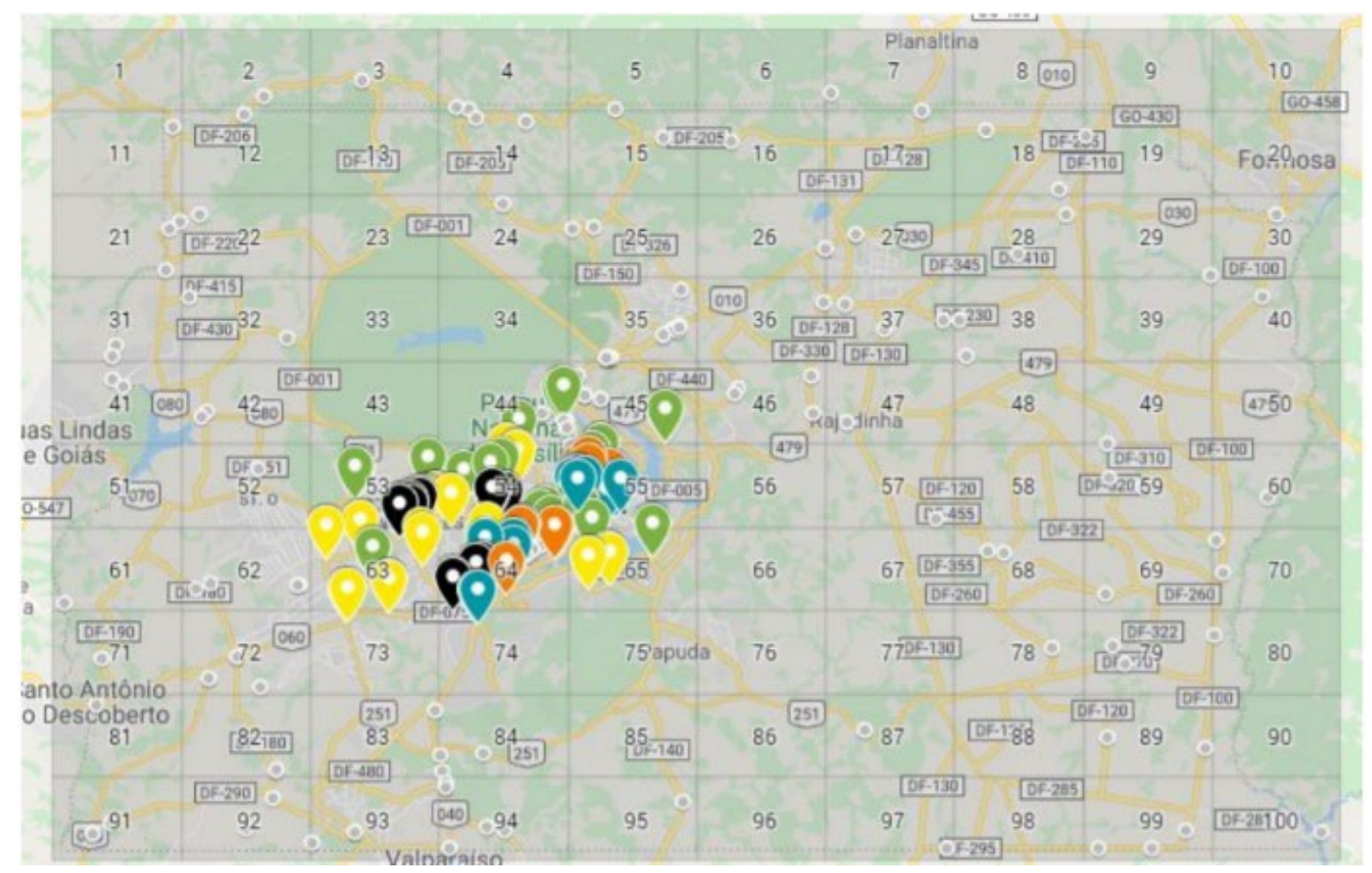

Figura 2 - Divisão dá Área do Distrito Federal e das OAEs em Malha 10 x 10

No ano de 2020 foi realizado um levantamento de todos os viadutos que seriam inspecionados no Distrito Federal, priorizando o Plano Piloto de Brasília, com isso, foram cadastrados 356 OAEs sob responsabilidade do Governo do Distrito Federal. Assim, para a aplicação da metodologia GDE/UnB, foram selecionados 168 das 356 OAEs cadastradas. Foi necessário a criação de um código de identificação para cada OAE, a fim de poder distribuí-las entre a equipe de trabalho e para que essas pudessem ser localizadas geograficamente. Tal código de identificação é composto na forma AAA_BBB_CCC_DDD_E, onde:

- AAA = Número da Malha;

- BBB = São os $2^{\circ}, 3^{\circ}$ e $4^{\circ}$ dígitos da longitude - para ordenar dentro da malha da esquerda para direita;

- CC = São os 2 últimos dígitos da longitude - insere a precisão necessária para distinguir pontos próximos horizontalmente;

- DD = São os 2 últimos dígitos da latitude - insere a precisão necessária para distinguir os pontos próximos verticalmente;

- $\mathbf{E}=$ Identifica o tipo de OAE, sendo "P" para Pontes e "V" para Viadutos.

Ainda na Figura 2, observa-se no mapa as OAEs selecionadas, bem como a divisão feita entre a equipe de inspeções. Vale ressaltar que ainda existem várias OAEs, representadas pelos pontos de cor cinza, que não foram contemplados neste trabalho, podendo ser elencadas como forma de sugestão para inspeções futuras. 


\subsection{Procedimento de inspeção}

Para início das análises, foi necessário um período de alinhamento junto a equipe de trabalho para se poder calibrar a aplicação da metodologia GDE/UnB, sendo feitas reuniões e discussões a respeito das análises e conclusões de cada integrante. Após esse balizamento, deu-se início às inspeções visuais, feitas por pelo menos duas pessoas, sendo uma delas responsável pelo preenchimento da ficha de inspeção elaborada com os respectivos danos encontrados em cada elemento das OAEs e outro indivíduo responsável pelo registro fotográfico, com o intuito de compor o banco de imagens que tornaria possível a visualização e reavaliação dos danos. Posteriormente, colocou-se as informações em planilhas programadas de modo que se obtenha o grau de deterioração da estrutura como resposta final, formando o banco de dados do Sistema de Gestão de Obras de Arte Especiais do Distrito Federal, SIGOA-DF. É válido lembrar que algumas OAEs, ou parte delas, tiveram seu acesso impossibilitado, pois seriam necessários equipamentos mais sofisticados para vencer barreiras físicas e garantir a segurança dos responsáveis pela vistoria, prejudicando algumas inspeções. Além disso algumas das estruturas a serem inspecionadas não eram acessíveis sem autorização do órgão gestão responsável.

Para obter condições de avaliação e registro mais seguras e menos conturbadas pelo tráfego de veículos, as inspeções ocorreram normalmente em horários pouco movimentados, focado nos fins de semana, no turno da manhã ou começo da tarde. Foram utilizados capacetes, botas e coletes refletivos, em atendimento à segurança do trabalho. A ficha de inspeção foi utilizada para a identificação, quantificação e análise das manifestações patológicas encontradas. Utilizou-se câmera fotográfica digital para registrar e catalogar os danos evidenciados em famílias em cada OAE inspecionada.

\section{RESULTADOS}

As inspeções de todas as OAEs forneceram informações de 2107 elementos estruturais e também foram constatadas 4539 manifestações patológicas. As famílias de elementos consideradas foram tabuleiro, pilar, transversina, guarda rodas, cortina e longarina. As manifestações patológicas consideradas foram corrosão de armaduras, fissuras, cobrimento deficiente, desagregação, desplacamento, abrasão do concreto, manchas, infiltração de água, eflorescência, flechas, deslocamento por empuxo, falhas de concretagem e desvio construtivo de geometria.

Nesta seção serão apresentados alguns resultados quantitativos referentes aos dados coletados, além de uma breve análise acerca da OAE mais crítica inspecionada.

\subsection{Análise geral das OAEs inspecionadas}

Do total de OAEs cadastradas no Distrito Federal, 47,2\% foram inspecionadas e apresentadas neste trabalho, observando-se o valor do Grau de Deterioração (Gd) mais elevado de 150,84 (nível crítico). O menor valor de Gd calculado para as estruturas inspecionadas foi de 0,91, correspondente a um nível baixo de deterioração de acordo com os critérios estabelecidos por Euqueres (2011). O Grau de Deterioração médio das estruturas inspecionadas foi de 29,33, o que também corresponde a um nível de deterioração baixo pelos mesmos critérios. Os valores máximo, mínimo e médio calculados são apresentados na Tabela 1.

Tabela 1 - Panorama geral das OAEs levantadas e inspecionadas

\begin{tabular}{|c|c|c|}
\hline Descrição & Gd & Nível \\
\hline Gd Máximo & 150.84 & Crítico \\
\hline Gd Mínimo & 0.91 & Baixo \\
\hline Gd Médio & 29.33 & Médio \\
\hline
\end{tabular}


Considerando-se as 5 OAEs mais críticas, observa-se uma variação de $58 \%$ na comparação entre o primeiro e o quinto valor de Gd, sendo os quatro primeiras OAEs classificadas em nível crítico e a quinta em nível sofrível. As 5 OAEs menos críticas são todas classificadas em nível baixo, sendo a variação entre o primeiro e o quinto valor de aproximadamente $306 \%$. A relação das 5 OAEs mais críticas e das 5 OAEs menos críticas é apresentada na Tabela 2.

Tabela 2 - Relação das OAEs mais críticas e menos críticas inspecionadas

\begin{tabular}{|l|c|l|c|}
\hline \multicolumn{2}{|c|}{ 5 OAEs Mais Críticas } & \multicolumn{1}{c|}{ 5 OAEs Menos Críticas } \\
\hline \multicolumn{1}{|c|}{ OAE } & Gd & \multicolumn{1}{c|}{ OAE } & Gd \\
\hline $\begin{array}{l}\text { Viaduto 2 sobre acesso à Ponte Costa e } \\
\text { Silva }\end{array}$ & 150.84 & $\begin{array}{l}\text { Viaduto na DF-002 Conjunto } \\
\text { Nacional }\end{array}$ & 3.70 \\
\hline Viaduto 1 de acesso ao Guará & 142.84 & Viaduto na DF-002 final da Asa Sul 2 & 3.68 \\
\hline & 133.20 & $\begin{array}{l}\text { Viaduto na DF-002 em frente ao } \\
\text { CONIC }\end{array}$ & 1.87 \\
\hline Ponte 1 sobre Córrego Riacho Fundo & 120.70 & $\begin{array}{l}\text { Viaduto sobre a via S2 ao lado do } \\
\text { Prédio Gilberto Salomão }\end{array}$ & 1.26 \\
\hline $\begin{array}{l}\text { Viaduto sobre a DF-003 acesso SIA } \\
\text { Viaduto sob o Eixo Monumental } \\
\text { próximo à catedral militar 2 }\end{array}$ & 95.24 & $\begin{array}{l}\text { Viaduto na DF-002 Sul em frente ao } \\
\text { BRB 2 }\end{array}$ & 0.91 \\
\hline
\end{tabular}

A Tabela 3 expõe a classificação das 168 OAEs inspecionadas em relação aos níveis de deterioração, bem como a distribuição das mesmas nos intervalos definidos por Euqueres (2011). Observa-se que a maior parte das OAEs do Distrito Federal se encontram nos níveis baixo e médio, seguidos pelos níveis alto, sofrível e crítico, respectivamente.

Tabela 3 - Classificação das OAEs inspecionadas de acordo com o nível de deterioração

\begin{tabular}{|c|c|c|c|}
\hline $\begin{array}{c}\text { Nível de } \\
\text { Deterioração }\end{array}$ & Gd & OAEs & $\%$ \\
\hline Baixo & $0-15$ & 78 & $46.4 \%$ \\
\hline Médio & $15-50$ & 59 & $35.1 \%$ \\
\hline Alto & $50-80$ & 19 & $11.3 \%$ \\
\hline Sofrível & $80-100$ & 8 & $4.8 \%$ \\
\hline Crítico & $>100$ & 4 & $2.4 \%$ \\
\hline
\end{tabular}

Analisando-se as famílias dos elementos inspecionados, conforme exposto na Tabela 4, observase que as famílias de tabuleiros, pilares e guarda rodas são as de maior grau de deterioração médio. Ademais, a Tabela 5 mostra a quais famílias pertencem as 30 manifestações patológicas de maior intensidade encontradas, sendo as fissuras e a corrosão de armaduras em pilares os danos mais críticos constatados.

Tabela 4 - Análise das famílias de acordo com o Grau de Deterioração da Família médio

\begin{tabular}{|l|c|c|}
\hline \multicolumn{1}{|c|}{ Família } & $\begin{array}{c}\text { GDF } \\
\text { Médio }\end{array}$ & $\begin{array}{c}\text { Elementos } \\
\text { Inspecionados }\end{array}$ \\
\hline Tabuleiro & 36.51 & 555 \\
\hline Pilar & 25.25 & 966 \\
\hline Guarda Rodas & 23.68 & 326 \\
\hline Cortina & 22.10 & 88 \\
\hline
\end{tabular}




\begin{tabular}{|l|l|l|} 
Transversina & 18.20 & 76 \\
\hline Longarina & 16.72 & 85 \\
\hline Cortina Central & 11.61 & 11 \\
\hline
\end{tabular}

Tabela 5 - Famílias das 30 manifestações patológicas de maior intensidade

\begin{tabular}{|l|c|c|c|c|c|c|}
\hline \multicolumn{1}{|c|}{ Dano / Família } & TOTAL & Cortina & Guarda Rodas & Pilar & Tabuleiro & Transversina \\
\hline Corrosão de armaduras & 9 & 0 & 1 & 5 & 2 & 1 \\
\hline Fissuras & 15 & 2 & 2 & 11 & 0 & 0 \\
\hline Cobrimento deficiente & 1 & 0 & 0 & 0 & 1 & 0 \\
\hline Desagregação & 3 & 0 & 0 & 3 & 0 & 0 \\
\hline Desplacamento & 2 & 0 & 0 & 0 & 1 & 1 \\
\hline
\end{tabular}

Por fim, pode-se listar as manifestações patológicas mais comumente encontradas, expostas na Figura 4. Observa-se que os danos mais recorrentes são as manchas (24,59\%), seguidas da infiltração de água $(13,20 \%)$, da eflorescência $(13,10 \%)$ e da corrosão de armaduras $(11,03 \%)$.

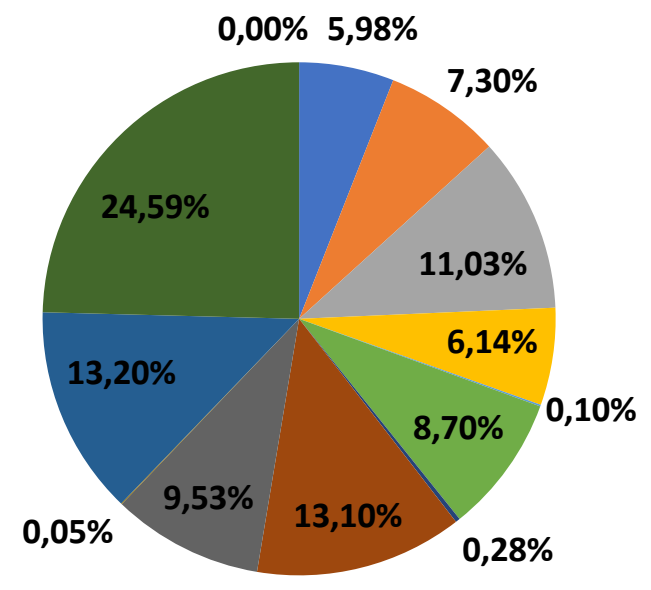

Abrasão do concreto

Cobrimento deficiente

Corrosão de armaduras

Desagregação

Deslocamento por empuxo

Desplacamento

Desvio de geometria

Eflorescência

Falhas de concretagem

Flechas

Infiltração de água

Manchas

Sinais de Esmagamento

Figura 4 - Recorrência das manifestações patológicas

\subsection{Análise da OAE mais crítica}

A OAE mais crítica dentre as vistoriadas foi o Viaduto 2 sobre acesso à Ponte Costa e Silva, cujo código é 064_789_22_89_V. Para sua vistoria, a estrutura foi dividida entre os seguintes elementos: 2 guarda-rodas, 6 pilares e 4 tabuleiros. As Tabelas 6,7 e 8 exemplificam as planilhas utilizadas para a inspeção de cada família de elemento do viaduto em questão, demonstrando os danos e seus fatores de ponderação, além do fator de intensidade que foi atribuído após a avaliação, e, por fim, o resultado do grau de deterioração de elemento (Gde). 
Tabela 6 - Grau de deterioração do Guarda-Rodas Leste

\begin{tabular}{|c|c|c|c|c|c|c|}
\hline Elemento & $\mathrm{Fr}$ & Dano & $\mathrm{Fp}$ & $\mathbf{F i}$ & D & Gde \\
\hline \multirow{11}{*}{ Guarda Rodas Leste } & \multirow{11}{*}{$\begin{array}{l}\text { ㄱ } \\
\text { II } \\
\text { L }\end{array}$} & Cobrimento deficiente & 3 & 3 & 24 & \multirow{11}{*}{34,67} \\
\hline & & Corrosão de armaduras & 5 & 2 & 8 & \\
\hline & & Desagregação & 3 & 1 & 2,4 & \\
\hline & & Desplacamento & 3 & 2 & 4,8 & \\
\hline & & Eflorescência & 2 & & 0 & \\
\hline & & Falhas de concretagem & 2 & & 0 & \\
\hline & & Fissuras (2 a 5) & 2 & 1 & 1,6 & \\
\hline & & Manchas & 3 & 1 & 2,4 & \\
\hline & & Sinais de Esmagamento & 4 & & 0 & \\
\hline & & Infiltração de água & 3 & & 0 & \\
\hline & & Abrasão do Concreto & 3 & & 0 & \\
\hline
\end{tabular}

Tabela 7 - Grau de deterioração do Pilar Norte-Oeste

\begin{tabular}{|c|c|c|c|c|c|c|}
\hline Elemento & $\mathrm{Fr}$ & Dano & $\mathrm{Fp}$ & $\mathbf{F i}$ & D & Gde \\
\hline \multirow{13}{*}{ Pilar Norte-Oeste } & \multirow{13}{*}{$\begin{array}{l}n \\
\text { II } \\
\text { L }\end{array}$} & Cobrimento deficiente & 3 & & 0 & \multirow{13}{*}{146,58} \\
\hline & & Corrosão de armaduras & 5 & 4 & 100 & \\
\hline & & Desagregação & 3 & 4 & 60 & \\
\hline & & Deslocamento por empuxo & 5 & & 0 & \\
\hline & & Desplacamento & 3 & 3 & 24 & \\
\hline & & Desvio de geometria & 3 & & 0 & \\
\hline & & Eflorescência & 2 & & 0 & \\
\hline & & Falhas de concretagem & 2 & 2 & 3,2 & \\
\hline & & Fissuras (2 a 5) & 2 & & 0 & \\
\hline & & Infiltração de água & 3 & & 0 & \\
\hline & & Manchas & 3 & & 0 & \\
\hline & & Sinais de Esmagamento & 4 & & 0 & \\
\hline & & Abrasão do concreto & 3 & & 0 & \\
\hline
\end{tabular}

Tabela 8 - Grau de deterioração do Tabuleiro Centro-Norte

\begin{tabular}{|c|c|c|c|c|c|c|}
\hline Elemento & $\mathrm{Fr}$ & Dano & $\mathrm{Fp}$ & $\mathrm{Fi}$ & D & Gde \\
\hline \multirow{11}{*}{ Tabuleiro Centro-Norte } & \multirow{11}{*}{$\begin{array}{l}+ \\
\text { II } \\
\stackrel{4}{4}\end{array}$} & Cobrimento deficiente & 3 & 1 & 2,4 & \multirow{11}{*}{62,14} \\
\hline & & Corrosão de armaduras & 5 & 3 & 40 & \\
\hline & & Desagregação & 3 & & 0 & \\
\hline & & Desplacamento & 3 & 1 & 2,4 & \\
\hline & & Eflorescência & 2 & 4 & 40 & \\
\hline & & Falhas de concretagem & 2 & & 0 & \\
\hline & & Fissuras (2 a 5) & 2 & & 0 & \\
\hline & & Flechas & 5 & & 0 & \\
\hline & & Infiltração de água & 3 & 1 & 2,4 & \\
\hline & & Manchas & 3 & 1 & 2,4 & \\
\hline & & Abrasão do concreto & 3 & & 0 & \\
\hline
\end{tabular}

A partir dos valores do Gde, é possível fazer o cálculo do grau de deterioração das famílias (Gdf) e, por fim, o cálculo do grau de deterioração da estrutura $(\mathrm{Gd})$. A Tabela 9 resume o resultado de todos os índices para todos os elementos. 
Tabela 9 - Resultados obtidos para o Viaduto 064_789_22_89_V

\begin{tabular}{|c|c|c|c|c|c|}
\hline Elemento & $\begin{array}{c}\text { Grau de } \\
\text { Deterioração } \\
\text { do Elemento } \\
\text { (Gde) }\end{array}$ & $\begin{array}{c}\text { Grau de } \\
\text { Deterioração } \\
\text { da Família } \\
\text { (Gdf) }\end{array}$ & $\begin{array}{l}\text { Fator de } \\
\text { Relevância } \\
\text { (Fr) }\end{array}$ & $\begin{array}{c}\text { Grau de } \\
\text { Deterioração } \\
\text { Estrutural } \\
\text { (Gd) }\end{array}$ & $\begin{array}{c}\text { Nível de } \\
\text { Deterioração } \\
\text { da Estrutura }\end{array}$ \\
\hline Guarda Rodas Leste & 34,67 & \multirow{2}{*}{39,19} & \multirow{2}{*}{1} & \multirow{12}{*}{150,84} & \multirow{12}{*}{ Crítico } \\
\hline Guarda Rodas Oeste & 13,33 & & & & \\
\hline Pilar Sul-Leste & 145,65 & \multirow{6}{*}{190,37} & \multirow{6}{*}{5} & & \\
\hline Pilar Sul-Oeste & 146,12 & & & & \\
\hline Pilar Centro-Leste & 0,00 & & & & \\
\hline Pilar Centro-Oeste & 1,60 & & & & \\
\hline Pilar Norte-Leste & 28,00 & & & & \\
\hline Pilar Norte-Oeste & 146,58 & & & & \\
\hline Tabuleiro Sul & 12,67 & \multirow{4}{*}{71,75} & \multirow{4}{*}{4} & & \\
\hline Tabuleiro Centro-Sul & 13,24 & & & & \\
\hline Tabuleiro Centro-Norte & 62,14 & & & & \\
\hline Tabuleiro Norte & 5,12 & & & & \\
\hline
\end{tabular}

Observa-se a partir da tabela que a família dos pilares obteve Gdf crítico, pois estão acima do valor 100 , tendo recomendação de intervenção imediata. Uma das causas para isso é o elemento Pilar Norte-Oeste, que obteve o maior Gde da estrutura, devido à grande perda na seção de armaduras expostas, sendo considerada a manifestação patológica do tipo corrosão de armadura com Fator de Intensidade (Fi) igual a 4, além de dano por desagregação, desplacamento e falhas de concretagem. A Figura 5 expõe o nível de deterioração em um dos elementos de pilar inspecionados.

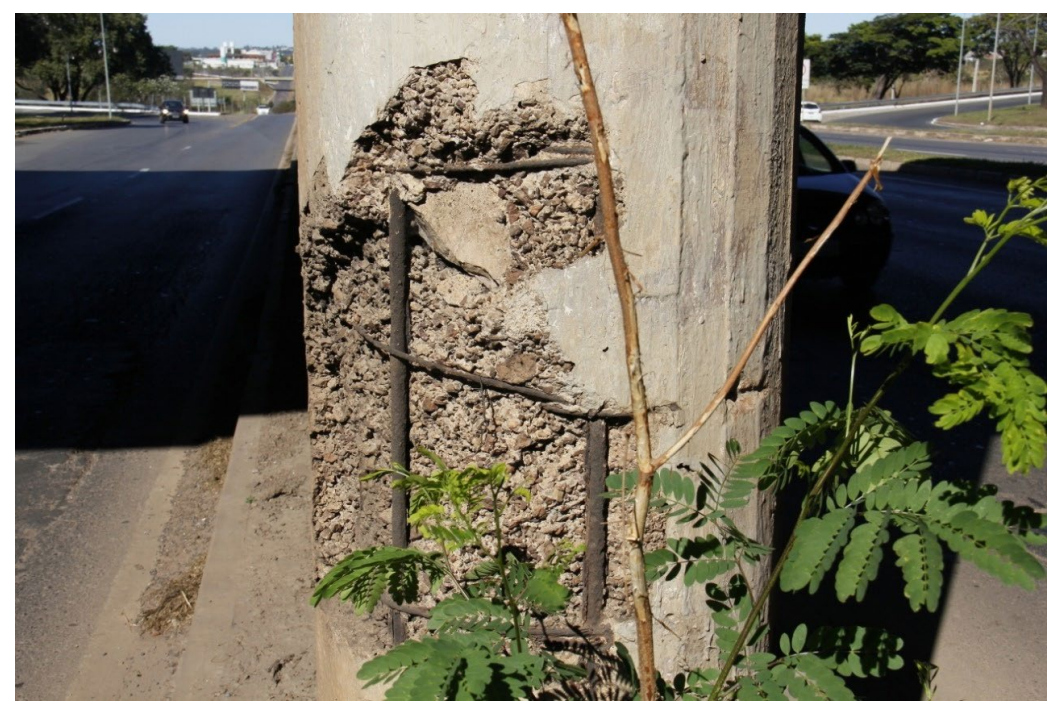

Figura 5 - Elemento pilar com corrosão de armadura e desplacamento

Ademais, foram observados em 3 dos 4 tabuleiros deterioração por eflorescência e por corrosão de armadura, sendo o Tabuleiro Centro-Norte avaliado como em pior situação. A Figura 6 representa a intensidade do dano eflorescência para os tabuleiros. 


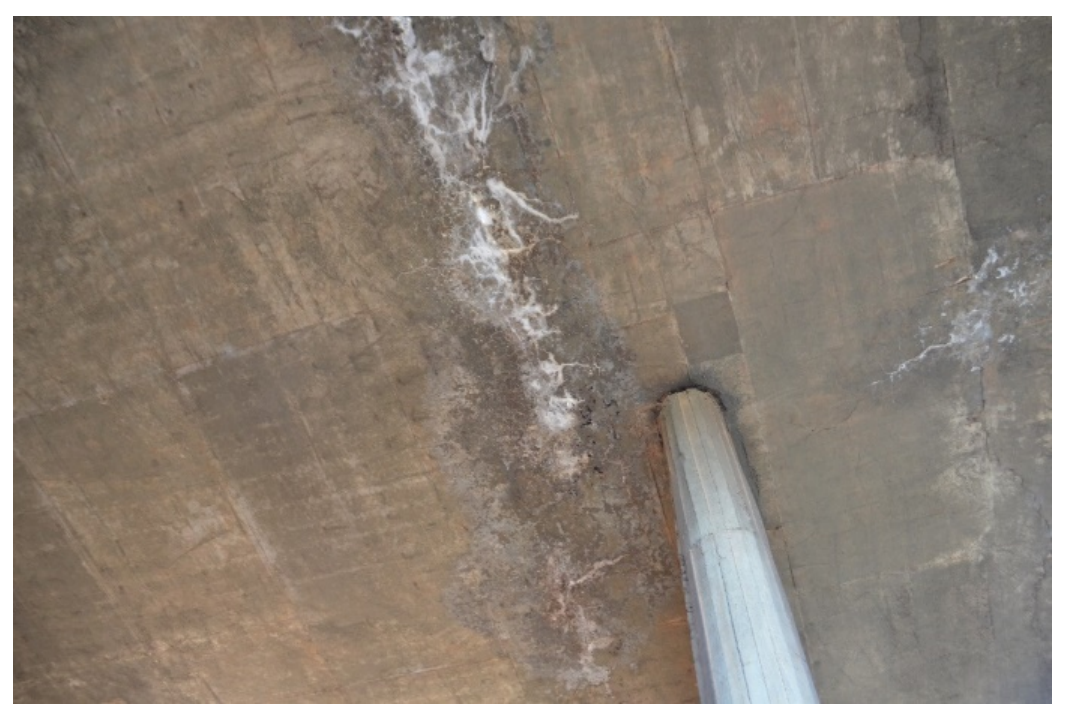

Figura 6 - Elemento tabuleiro com eflorescência

Nota-se que a família dos guarda-rodas é a que possui o melhor estado de conservação da estrutura, com Gdf igual a 39,19. Fazendo o cálculo do grau de deterioração do viaduto, obteve-se um valor de Gd igual a 150,84, o que está compreendido pelo nível crítico, obtendo a recomendação de intervenção imediata de acordo com Euqueres (2011).

\section{CONCLUSÕES}

Para o presente trabalho aplicou-se a metodologia do Grau de Deterioração de Estruturas desenvolvida pela Universidade de Brasília (GDE/UnB) em OAEs de Brasília-DF.

O menor valor de Gd calculado para as estruturas inspecionadas foi de 0,91 , correspondente a um nível baixo de deterioração de acordo com os critérios estabelecidos por Euqueres (2011). O Grau de Deterioração médio das estruturas inspecionadas foi de 29,33, o que também corresponde a um nível de deterioração baixo pelos mesmos critérios. A OAE mais crítica dentre as vistoriadas foi o Viaduto 2 sobre acesso à Ponte Costa e Silva (código 064_789_22_89_V), com um valor de Gd igual a 150,84, o que está compreendido pelo nível crítico, obtendo a recomendação de intervenção imediata. Do estudo, observa-se que a maior parte das OAEs estão nos níveis baixo e médio de deterioração, seguidos dos elementos com níveis alto, sofrível e crítico, respectivamente. Além disso, percebeu-se que os danos mais recorrentes são as manchas, infiltração de água, eflorescência e corrosão de armaduras.

O estudo demonstrou a aplicabilidade de um método eficiente, capaz de facilitar e simplificar a periodicidade de inspeções em OAEs, permitindo que se compreenda o funcionamento e propagação dos danos com o tempo. Por fim, a aplicação da metodologia GDE/UnB permite estipular um período máximo para intervenção.

Por fim, os resultados obtidos no presente trabalho mostram um panorama geral do estado de conservação de praticamente metade das OAEs levantadas no Distrito Federal, algumas das quais em situação crítica e cuja manutenção não deve ser negligenciada, exigindo intervenção imediata, indicando sinal máximo de alerta. Busca-se, dessa forma, auxiliar na tomada de decisões que evitem acidentes e otimizem a vida útil dessas estruturas essenciais para um bom fluxo da mobilidade urbana.

\section{AGRADECIMENTOS}


À Universidade de Brasília, essencial no processo de formação e aprendizado do conteúdo e habilidades necessárias para a elaboração deste e outros trabalhos.

Ao Grupo de Estudos de Tecnologias da Engenharia Civil da Universidade de Brasília - GETECUnB.

À comissão organizadora do CONPAT 2021, pela oportunidade da submissão deste trabalho.

\section{REFERÊNCIAS}

Boldo, P. (2002), Análise Quantitativa de Estruturas de Concreto Armado de Edificações no Âmbito do Exército Brasileiro. Dissertação de Mestrado, Publicação E.DM-001A/02, Departamento de Engenharia Civil e Ambiental, Universidade de Brasília, Brasília, DF, 295 p.

Castro, E. K. (1994), Desenvolvimento de Metodologia para Manutenção de Estruturas de Concreto Armado. Dissertação de Mestrado, Publicação No: E.DM-004A/94, Departamento de Engenharia Civil, Universidade de Brasília, Brasília, DF, 155 p.

Chaves, L. (2020) Avaliação de Obras de Arte Especiais da cidade de Brasília pela Metodologia do Grau de Deterioração Estrutural da Universidade de Brasília. UnB

Euqueres, P.; (2011). Metodologia de Inspeção em Estruturas de Pontes de Concreto Armado. Dissertação de Mestrado, Escola de Engenharia Civil, Universidade Federal de Goiás, Goiânia, GO, $168 \mathrm{p}$.

Fonseca, R. P. (2007). A Estrutura do Instituto Central de Ciências: Aspectos Históricos, Científicos e Tecnológicos de Projeto, Execução, Intervenções e Propostas de Manutenção. Dissertação de Mestrado em Estruturas e Construção Civil, Publicação E.DM - 006 A/07, Departamento de Engenharia Civil e Ambiental, Universidade de Brasília, Brasília, DF, 213p.

Klein, D., Gastal F., Campanolo, J.L \& Silva Filho, L. C. (1991). "Critérios adotados na vistoria e avaliação de obras de arte", XXV Jornada Sul Americana de Engenharia Estrutural, Porto Alegre, pp.185-196, novembro.

Lauria, M. L. M. (2018) Avaliação dos Viadutos do Eixo Rodoviário Sul de Brasília pela Metodologia GDE/UnB. 2018. Trabalho de Conclusão de Curso (Bacharelado em Engenharia Civil) - Universidade de Brasília,

Lopes, B. A. R. (1998), "Sistema de Manutenção Predial para Grades Estoques de Edifícios: Estudo para inclusão do componente "Estrutura de Concreto". Dissertação de Mestrado, Publicado No: E.DM-011A/98, Departamento de Engenharia Civil, Universidade de Brasília, Brasília, DF, 308p.

Mitre, M. P. (2005); Metodologia para inspeção e diagnóstico de pontes e viadutos de concreto / M. P. Mitre - São Paulo, 148p.

Pavoni, F. B. (2019) Avaliação das Obras de Arte Especiais do Eixo Rodoviário Norte de Brasília pela Metodologia do Grau de Deterioração Estrutural da Universidade de Brasília. 2019.

Trabalho de Conclusão de Curso (Bacharelado em Engenharia Civil) - Universidade de Brasília, [S. 1.]. 
Pereira, M. S. (2020), Avaliação de Obras de Arte Especiais da cidade de Brasília (lote 1) pela Metodologia do Grau de Deterioração Estrutural da Universidade de Brasília. UnB

Saraiva, C. (2020), Avaliação de Obras de Arte Especiais da cidade de Brasília pela Metodologia do Grau de Deterioração Estrutural da Universidade de Brasília. UnB

Verly, R. C. (2015). Avaliação de Metodologias de Inspeção como Instrumento de Priorização de Intervenções em Obras de Arte Especiais. Dissertação de Mestrado em Estruturas e Construção Civil, Publicação E.DM-015A/15, Departamento de Engenharia Civil e Ambiental, Universidade de Brasília, Brasília, DF, 178p. 